\title{
Utilizing social media as a platform to facilitate primary care physicians' exercise referrals among cancer patients and the general public
}

\section{Negin Niksirat and Parna Niksirat}

\section{University of British Columbia}

Delivery of primary care physicians' exercise referrals via social networking sites has not been established among cancer patients and the general public. Social media can be utilized as a platform for fostering improvements in following up with exercise and physical activity referrals. The benefits of utilizing such a mechanism include developing self-monitoring behaviours, self-efficacy, and social support among caner patients and the general public. A randomized trial using a Facebook-based physical activity intervention has proven feasible in the promotion of physical activity among young cancer survivors. ${ }^{1}$ Thus, as a delivery channel, social networking sites may hold potential for promoting physical activity and exercise among cancer patients and the general public. ${ }^{1}$

The benefits of physical activity and exercise as they relate to cancer prevention and side-effect treatment are well established. ${ }^{2,3}$ Research has shown that the protective and anti-inflammatory effects of physical exercise lower the risk of developing both colon cancer and postmenopausal breast cancer. ${ }^{3}$ However, physical activity participation has been shown to decrease in colorectal cancer survivors over the course of chemotherapy. ${ }^{4}$ In addition, compared to pre-diagnosis, there was a significant reduction in the percentage of colon cancer survivors meeting the American College of Sports Medicine guidelines during chemotherapy treatment. In this context, social media has the potential to increase patient physical activity by enhancing primary care physicians' exercise referrals.

Emerging observational evidence suggests that being physically active following diagnosis can lead to reduced risk of cancer recurrence and improved survival. ${ }^{4}$ Specifically, in comparison to the inactive group (<3 MET- hours/week), significantly improved disease- and recurrence-free survival was reported among those who engaged in 18-26.9 METhours/week after stage III colon cancer diagnosis.. ${ }^{4}$ Thus, incorporating an exercise-focused lifestyle might prevent cancer recurrence and improve quality of life. It is also believed that as part of cancer prevention efforts, the healthy population should be encouraged to participate in regular physical activity.

Researchers have attracted the interest of primary care physicians and rehabilitation professionals in disseminating health information. Primary care physicians are in regular interaction with large proportions of the population. ${ }^{5}$ Thus, the integration of novel and effective strategies that use social networking sites may prove beneficial in promoting active behaviours and improving fitness levels. ${ }^{6}$ This may also help enhance the delivery of exercise referrals and health communication between sport medicine professionals, the general public, and cancer patients. A social networking application or website could be developed with the aim of improving individuals' ability to participate in shared decision making about physical activity options.

A key setting for promoting physical activity is the primary health care arena. Although there is much evidence supporting the therapeutic value of physical activity and its role in lowering the risk of cancer, general practitioners must also contribute to taking initiatives to implement immediate exercise prescription in general practice. ${ }^{6}$ However, no official Canadian website or exercise referral programs currently exist. There is no platform for primary care physicians to connect cancer patients and the general public to exercise professionals who have a wide range of expertise in chronic disease management.

It has been shown that primary care physicians' messages and advice greatly influence the motivation of exerciserelated health behaviours among the general public. ${ }^{5-9}$ As a means of facilitating the writing of prescriptions for exercise, social media could enable primary health care workers to implement evidence-based treatment among the general public. The compliance of patients in relation 
C to exercise interventions could be supported by a social platform, like Facebook, to facilitate the adaptation of an active lifestyle by incorporating exercise and physical activity as part of daily routines. The implications for cancer patients and the general public include social accountability from having them publish their exercise goals as well as their self-monitoring behaviours online. As a result, they would be able to receive their physicians' advice with follow-up counseling.

Exercise can be used as means of cancer prevention and treatment among the general public and those already diagnosed with the disease. Consequently, it is crucial to promote the uptake of physically active behaviours in the home and community settings by targeting primary care physicians to encourage the general public and those undergoing cancer treatments to seek exercise prescriptions. A social media platform represents an ideal mechanism to accelerate the communication between primary care physicians, cancer patients, and the general public. Social media can be used by patients to help communicate with their primary health care physicians, thus bridging the gap between research and practice as it relates to increasing exercise referral. In this way, physicians will be positioned to deliver essential referrals for treating those at-risk for cancer in addition to training the disease-free population for appropriate usage of available community exercise resources and exercise activities.

\section{References}

1. Valle CG, Tate DF, Mayer DK, Allicock M, Cai J. A randomized trial of a Facebook-based physical activity intervention for young adult cancer survivors. Journal of Cancer Survivorship 2013:1-14

2. Gleeson M, Bishop NC, Stensel DJ, Lindley MR, Mastana SS, Nimmo MA. The anti-inflammatory effects of exercise: mechanisms and implications for the prevention and treatment of disease. Nature Reviews Immunology 2011;11(9):607-615.

3. Thune I, Brenn T, Lund E, Gaard M. Physical activity and the risk of breast cancer. N Engl J Med 1997;336(18):1269-1275.

4. Chung JY, Lee DH, Park J, Lee MK, Kang D, Min J, et al. Patterns of physical activity participation across the cancer trajectory in colorectal cancer survivors. Supportive Care in Cancer 2013;21(6):1605-1612.

5. Petrella RJ, Koval JJ, Cunningham DA, Paterson DH. Can primary care doctors prescribe exercise to improve fitness?: The step test exercise prescription (STEP) project. Am J Prev Med 2003;24(4):316-322.

6. Pavey T, Taylor A, Fox K, Hillsdon M, Anokye N, Campbell J, et al. Effect of exercise referral schemes in primary care on physical activity and improving health outcomes: systematic review and meta-analysis. BMJ: British Medical Journal 2011;343.

7. Williams NH, Hendry M, France B, Lewis R, Wilkinson C. Effectiveness of exercise-referral schemes to promote physical activity in adults: systematic review. British Journal of General Practice 2007;57(545):979-986.

8. Taylor $\mathrm{AH}$, Doust J, Webborn N. Randomised controlled trial to examine the effects of a GP exercise referral programme in Hailsham, East Sussex, on modifiable coronary heart disease risk factors. J Epidemiol Community Health 1998 Sep;52(9):595-601.

9. Sorensen J, Sorensen JB, Skovgaard T, Bredahl T, Puggaard L. Exercise on prescription: changes in physical activity and health-related quality of life in five Danish programmes. Eur J Public Health 2011 Feb;21(1):56-62.

\section{Negin Niksirat}

Negin Niksirat is in Rehabilitation and Oncology graduate program at University of British Columbia. Her area of research focuses on the chemotherapy-associated cognitive and physical dysfunction among colon cancer patients.

\section{Parna Niksirat}

Parna Niksirat is in Electronics graduate program at Simon Fraser University. Parna's research focuses on quadrotor helicopter and she has experience developing website. 